\title{
Glenoid fossa position in skeletal class ii malocclusion due to retrognathic mandible and skeletal class i malocclusion in pakistani population
}

\author{
Rabia Tabassuma ${ }^{a}$ Nazish Amjad ${ }^{b}$, Faiza Malik ${ }^{c}$ \\ ${ }^{a}$ Consultant, Department of Orthodontics, Madinah Teaching Hospital Faisalabad. \\ ${ }^{\mathrm{b}}$ Assistant Professor, Department of Orthodontics, Dental College HITEC-IMS, Taxila. \\ 'Assistant Professor, Head Department of Orthodontics, Sharif Medical and Dental College, Lahore. \\ *Corresponding Author's e-mail: drtabassum86@hotmail.com
}

\begin{abstract}
BACKGROUND \& OBJECTIVE: Different dental and skeletal abnormalities interact with each other to cause multiple malocclusions of various areas of the dentofacial region. Association of the mandible and cranial base influence the malocclusions in anteroposterior and vertical dimensions that influences the evaluation of the skeletal components of a particular patient, so the relationship of glenoid fossa to adjacent craniofacial components must be taken into account. This research is conducted for the comparison of glenoid fossa position in subjects presenting with class II skeletal malocclusion due to retrognathic mandible and class I skeletal malocclusion.

METHODOLOGY: Standardized lateral cephalograms of 130 patients were selected according to inclusion criteria from the orthodontic department. These radiographs were traced, and different angular and linear measurements were recorded. Then the position of glenoid fossa was compared in subjects of class II malocclusion with retrognathic mandible and class I malocclusion. SPSS version 17 was used for data analysis. Cephalometric measurements were analyzed, and glenoid fossa position was compared in both groups by using student's t-test. Statistical level of significance using student $t$ test was $p<$ 0.05
\end{abstract}

RESULTS: Position of glenoid fossa in subjects with class II malocclusion is more distal and posterior as compared to the subjects with class I malocclusion. The effective parameters for the measurements of glenoid fossa are GF-S on FH, GF-Ptm on FH, and GF-FMN with p value 0.001 .

CONCLUSION: A distally and posteriorly placed glenoid fossa is an important diagnostic feature of Class II skeletal malocclusion with the retrognathic mandible.

KEYWORDS: Glenoid fossa, Cranial base, Short mandible.

\section{INTRODUCTION}

One of the most complex joint of the human body is temoporomandibular joint which has a strong association with dental occlusion and skeletal relationships [1]. Genetics has an important role in the shape and structure of the temporomandibular joint but development of dental occlusion and mastication can influence the growth of temporomandibular joint and remodeling of surrounding structures. The mandibular condylar cartilage is secondary cartilage which is a growth site of the mandible, and it also assists in the articulation of the mandible with the glenoid fossa. The relationship of the temporomandibular joint with adjacent bones is maintained by adaptive growth at condylar cartilage ${ }^{[2]}$. Glenoid fossa is a structure which is present between the condyle of the mandible and temporal bone of the cranial base, that's way, its position can influence the relationship of the mandible with the cranium ${ }^{[3]}$.
Moreover, the development of both sagittal and vertical skeletal malocclusions of the jaws is frequently influenced by the correlation of mandible to the cranial base, so it is logical to assume that the glenoid fossa has a significant contribution in the development of various craniofacial regions. Class II skeletal malocclusion with retrognathic mandible can either be due to inaccurate jaw size or improper jaw position. The exact etiology can easily be diagnosed by orthodontist by evaluating the precise position of the glenoid fossa in the cranium. In this way, the proper treatment plan can be formulated ${ }^{[4]}$.

According to the results of previous researches, it is concluded that the position of glenoid fossa within cranium is more posterior in patients presenting with class II skeletal malocclusion in contrast to the subjects presenting with class I and class III skeletal malocclusions, in which it is more anteriorly located ${ }^{[5]}$.

Tabassum R, Amjad N, Malik F. Glenoid fossa position in skeletal class II malocclusion due to retrognathic mandible and skeletal class I malocclusion in Pakistani population. Journal of University Medical \& Dental College. 2021; 12(4):252-256. 
In contrary, in the patients presenting with low angle vertical proportions, the glenoid fossa was located more posterior in contrast to the subjects presenting with normal or high angle malocclusion ${ }^{[6]}$. According to the previous literature, the average value of GF-S on FH was $10.9 \mathrm{~mm}$, the average value of GF-Ptm on FH was $29.7 \mathrm{~mm}$ and the average value of GF-FMN was $67.6 \mathrm{~mm}$ for the Skeletal class I group as compared to $12.5 \mathrm{~mm}, 31.4 \mathrm{~mm}$ and $71 \mathrm{~mm}$ for Skeletal class I group respectively. According to these values, it is evident that glenoid fossa is located more distally in subjects presenting with class II skeletal malocclusion with retrognathic mandible ${ }^{[7]}$.

According to these reports, it is evident that the skeletal problem of the mandible can be enhanced by the glenoid fossa position, which can take part in the etiology of different malocclusion. It is a well-known perception that the proper functioning of temporomandibular joint is dependent upon the accurate orientation of mandibular condyle, articular disc, and the glenoid fossa. During the mandibular growth and displacement, remodeling of the glenoid fossa and condylar cartilage continues to occur to preserve the accurate positional correlation of both ${ }^{[7]}$.

Different dental and skeletal abnormalities interact with each other to cause multiple malocclusions of various areas of dentofacial region. Association of mandible and cranial base influence the malocclusions in anteroposterior and vertical dimensions, that's a way for the evaluation of the skeletal components of a particular patient, the relationship of glenoid fossa to adjacent craniofacial components must be taken into account ${ }^{[7,8]}$.

The purpose of this study is to clearly highlight the significance of glenoid fossa location in class II skeletal malocclusion due to the retrognathic mandible, which can then be corrected by functional jaw orthopedics. This research will be the first of its kind to be conducted in Pakistan. Earlier, Glenoid fossa position in class I and class II malocclusion was studied, but the quantification of retrognathic mandible in class II malocclusion was not done. This study will not only provide a population specific database for glenoid fossa position in Pakistani population but will also guide the treatment planning in class II malocclusion with retrognathic mandible. Dentofacial orthopedics can induce structural changes in the posterior wall of glenoid fossa in such subjects which will further contribute to the correction of such skeletal malocclusions.

\section{METHODOLOGY}

Approval for this research was taken from ethical committee of 28 Military Dental Centre Lahore. This was a cross sectional study which was conducted in Sharif Medical and Dental college in 6 months.

Pretreatment lateral cephalograms of 130 patients according to non- probability purposive sampling technique were selected from orthodontic department having following characteristics

- Normal vertical proportions

Age range from 12 to 25 years
- Class II skeletal malocclusion with retrognathic mandible

- Class I skeletal malocclusion.

- Patient with following characteristics were excluded from the study

- Class III Skeletal malocclusion

- Class II skeletal malocclusion with prognathic maxilla

- High vertical proportions

- Low vertical proportions

- History of trauma

- Complex craniofacial deformities or syndromes

All lateral cephalometric radiographs were traced and different angular and linear measurements were recorded (Figure-I ${ }^{[7]}$. Then the position of glenoid fossa was compared in subjects of class II malocclusion with retrognathic mandible and class I malocclusion. SPPS version 17 was used for data analysis. Cephalometric measurements were analyzed and glenoid fossa position was compared in both groups by using student's t test. Statistical level of significance using student $t$ test was $\mathrm{p}<0.05$

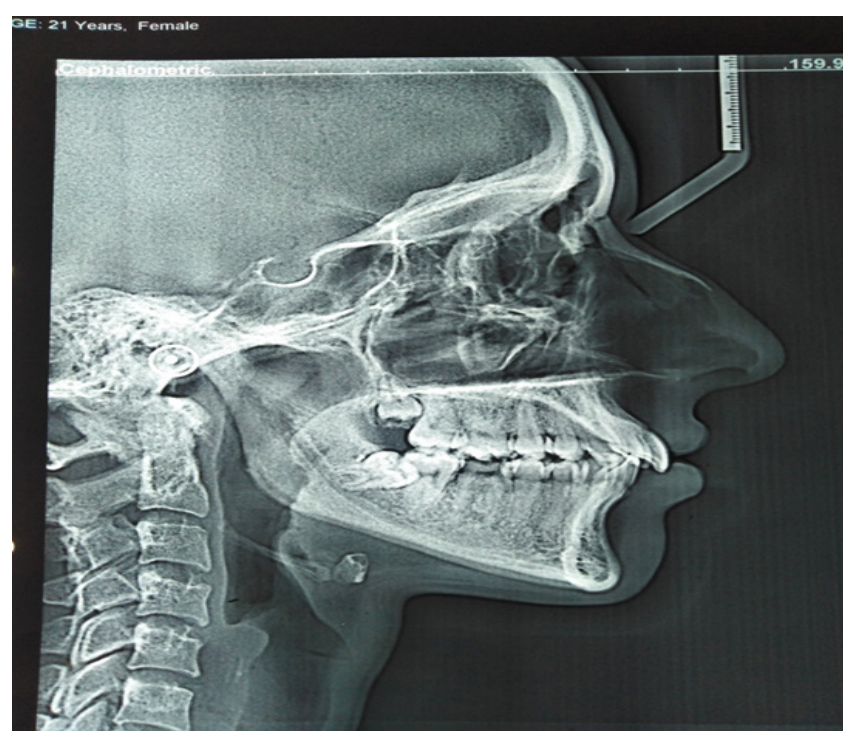

Figure-I: Lateral cephalogram.

RESULTS

SPSS version 17 was used for the analysis of Total data of 130 patients $(\mathrm{N}=130)$. Frequency of skeletal class I malocclusion was found to be 71 and skeletal class II malocclusion with retrognathic mandible was 59. Total patients were further distributed into two groups according to age. Frequency and percentage of patients were calculated in both groups Table-I. Distribution of gender was measured in both skeletal groups Table-II. Descriptive statistics of GF-S on FH, GF-Ptm on FH and GF-FMN were calculated in less than 18 years age group and more than 18 years age group and their comparison was done in both skeletal classes by using student's t test Table-III.

Descriptive statistics of GF-S on FH, GF-Ptm on FH and GF-FMN were calculated in males and females and their comparison was done in both skeletal classes by using student's t test Table-IV. 
Tabassum R, Amjad N, Malik F.

Table-I: Distribution of age in both skeletal classes.

\begin{tabular}{ccccc}
\hline & & \multicolumn{3}{c}{ Age Distribution } \\
\hline \multirow{3}{*}{ Class } & Skeletal class I & $47(51.6 \%)$ & $24(61.5 \%)$ & 71 \\
& & & & \\
& $\begin{array}{c}\text { Skeletal class II with } \\
\text { retrognathic mandible }\end{array}$ & $44(48.4 \%)$ & $15(38.5 \%)$ & 59 \\
Total & 91 & 39 & 130 \\
\hline
\end{tabular}

Table-II: Distribution of gender in both skeletal classes.

\begin{tabular}{ccccc}
\hline \multicolumn{4}{c}{ Gender } \\
\hline Class & Male & Female & Total \\
\hline \multirow{2}{*}{$\begin{array}{c}\text { Skeletal class I } \\
\text { with retrognathic } \\
\text { mandible }\end{array}$} & $24(49.0 \%)$ & $47(58.0 \%)$ & 71 \\
\hline Total & $25(51.0 \%)$ & $34(42.0 \%)$ & 59 \\
\hline
\end{tabular}

Table-III: Comparison of GF-S on FH, GF-Ptm on FH and GF-FMN in Skeletal class I and skeletal class II with retrognathic mandible in both age groups.

\begin{tabular}{|c|c|c|c|c|c|}
\hline Age Distribution & Variables & Class & $\mathbf{n}$ & Mean \pm SD & p-value \\
\hline \multirow{6}{*}{$\leq 18$ years } & GF-S on $\mathrm{FH}^{\mathrm{a}}$ & Skeletal class I & 47 & $10.128 \pm 0.8107$ & \multirow[b]{2}{*}{$<0.001$} \\
\hline & & Skeletal class II with retrognathic mandible & 44 & $12.159 \pm 3.0629$ & \\
\hline & \multirow[t]{2}{*}{ GF-Ptm on $\mathrm{FH}^{\mathrm{b}}$} & Skeletal class I & 47 & $28.532 \pm 1.3729$ & \multirow{2}{*}{$<0.001$} \\
\hline & & Skeletal class II with retrognathic mandible & 44 & $30.295 \pm 2.1521$ & \\
\hline & \multirow[t]{2}{*}{ GF-FMN ${ }^{c}$} & Skeletal class I & 47 & $76.787 \pm 1.4361$ & \\
\hline & & Skeletal class II with retrognathic mandible & 44 & $79.432 \pm 1.9577$ & $<0.001$ \\
\hline \multirow{6}{*}{$>18$ years } & \multirow[t]{2}{*}{ GF-S on $\mathrm{FH}^{\mathrm{a}}$} & Skeletal class I & 24 & $10.188 \pm 0.7344$ & \multirow[t]{2}{*}{0.021} \\
\hline & & Skeletal class II with retrognathic mandible & 15 & $11.133 \pm 1.3425$ & \\
\hline & \multirow[t]{2}{*}{ GF-Ptm on $\mathrm{FH}^{\mathrm{b}}$} & Skeletal class I & 24 & $28.708 \pm 1.3345$ & \multirow[t]{2}{*}{0.305} \\
\hline & & Skeletal class II with retrognathic mandible & 15 & $29.400 \pm 2.3161$ & \\
\hline & \multirow[t]{2}{*}{ GF-FMN ${ }^{c}$} & Skeletal class I & 24 & $76.833 \pm 1.2655$ & \multirow[t]{2}{*}{0.001} \\
\hline & & Skeletal class II with retrognathic mandible & 15 & $78.767 \pm 1.7099$ & \\
\hline
\end{tabular}

a) (Glenoid fossa to sella on Frankfort horizontal line, mm).

b) (Glenoid fossa to pterygomaxillary fissure on Frankfort horizontal line, mm).

c) (Glenoid fossa to frontomaxillonasal suture, $\mathrm{mm}$ ).

Table-IV: Comparison of GF-S on FH, GF-Ptm on FH and GF-FMN in Skeletal class I and Skeletal class II with retrognathic mandible in males and females.

\begin{tabular}{|c|c|c|c|c|c|}
\hline Gender & Variables & Class & $\mathrm{n}$ & Mean \pm SD & p-value \\
\hline \multirow{5}{*}{ Male } & GF-S on $\mathrm{FH}^{\mathrm{a}}$ & Skeletal class I & 24 & $10.104 \pm 0.7220$ & \multirow{4}{*}{$\begin{array}{l}0.007 \\
0.006\end{array}$} \\
\hline & & $\begin{array}{l}\text { Skeletal class II with retrognathic } \\
\text { mandible }\end{array}$ & 25 & $12.400 \pm 3.8487$ & \\
\hline & GF-Ptm on $\mathrm{FH}^{\mathrm{b}}$ & Skeletal class I & 24 & $28.479 \pm 1.5357$ & \\
\hline & & $\begin{array}{l}\text { Skeletal class II with retrognathic } \\
\text { mandible }\end{array}$ & 25 & $29.840 \pm 1.7424$ & \\
\hline & GF-FMN $^{c}$ & Skeletal class I & 24 & $76.875 \pm 1.2001$ & \multirow{3}{*}{$<0.001$} \\
\hline \multirow{7}{*}{ Female } & & $\begin{array}{l}\text { Skeletal class II with retrognathic } \\
\text { mandible }\end{array}$ & 25 & $79.280 \pm 2.0314$ & \\
\hline & GF-S on $\mathrm{FH}^{\mathrm{a}}$ & Skeletal class I & 47 & $10.170 \pm 0.8161$ & \\
\hline & & $\begin{array}{l}\text { Skeletal class II with retrognathic } \\
\text { mandible }\end{array}$ & 34 & $11.529 \pm 1.4972$ & \multirow[t]{2}{*}{$<0.001$} \\
\hline & GF-Ptm on $\mathrm{FH}^{\mathrm{b}}$ & Skeletal class I & 47 & $28.649 \pm 1.2637$ & \\
\hline & & $\begin{array}{l}\text { Skeletal class II with retrognathic } \\
\text { mandible }\end{array}$ & 34 & $30.235 \pm 2.5113$ & \multirow{3}{*}{$\begin{array}{l}<0.001 \\
<0.001\end{array}$} \\
\hline & GF-FMN ${ }^{c}$ & Skeletal class I & 47 & $76.766 \pm 1.4628$ & \\
\hline & & $\begin{array}{l}\text { Skeletal class II with retrognathic } \\
\text { mandible }\end{array}$ & 34 & $79.250 \pm 1.8392$ & \\
\hline
\end{tabular}

a) (Glenoid fossa to sella on Frankfort horizontal line, $\mathrm{mm}$ ).

b) (Glenoid fossa to pterygomaxillary fissure on Frankfort horizontal line, mm).

c) (Glenoid fossa to frontomaxillonasal suture, $\mathrm{mm}$ ). 


\section{DISCUSSION}

The glenoid fossa position has a significant contribution in the etiology of multiple orthodontic malocclusions. By evaluating the glenoid fossa position in the orthodontic diagnostic process, an important structure of the craniofacial area can be taken into account ${ }^{[3,4,9]}$.

The chief significance of evaluating the glenoid fossa location within cranium is in formulating the treatment plan of multiple malocclusions. Glenoid fossa location is a main contributor of various malocclusions in vertical and sagittal dimensions, that's way it is an important indicator of a certain form of malocclusion ${ }^{[10]}$.

By using the cephalometric radiographs for the evaluation of glenoid fossa position, patients presenting with class I skeletal malocclusion were found to have posteriorly located glenoid fossa as a part of their craniofacial features as compared to the patients presenting with class III malocclusion having anteriorly located glenoid fossa ${ }^{[7]}$.

The current study was conducted on 130 patients who fulfilled the inclusion criteria. Both male and female patients of 12 years to 25 years with normal vertical proportions were included in the study. Patients having history of trauma, severe craniofacial abnormalities and syndromes were excluded from the study.

In this study three measurements were taken for the evaluation of glenoid fossa which are the distance of glenoid fossa from sella (on Frankfort horizontal line, mm), from pterygomaxillary fissure (GF-Ptm on Frankfort horizontal line, $\mathrm{mm}$ ) and from frontomaxillonasal suture (GF-FMN, $\mathrm{mm})$. After that, the position of glenoid fossa was compared in subjects presenting with class II skeletal malocclusions due to retrognathic mandible and class I skeletal malocclusion.

The present study indicates that all these three measurements (GF-FMN, GF-S on FH and GF-Ptm on FH) are important for determining the difference of glenoid fossa position in both groups. This conclusion is different from the results of the study conducted by Giuntini et al ${ }^{[7]}$ according to that GFFMN is a more important measurement for the determination of glenoid fossa position.

The present study indicates that the glenoid fossa is located more posterior and distal in subject with class II skeletal malocclusion with retrognathic mandible as compared to the subjects with class I malocclusion.

The mean distance of glenoid fossa from frontomaxillonasal suture in the subjects of class II malocclusion was $2.5 \mathrm{~mm}$ larger than the same measurement in subjects of class I skeletal malocclusion. This difference is important from statistical as well as from clinical point of view, because it exhibits that, in the absence of dentofacial inconsistencies Class II skeletal malocclusion in the particular patient can be associated with a posterior position of the glenoid fossa with the result of a substantial mandibular retrognathism.

These conclusions verify the results of the previous study conducted by Droel \& Isaacson ${ }^{[10]}$ who also concluded that glenoid fossa was $2.5 \mathrm{~mm}$ distally displaced in the subjects with skeletal class II malocclusion as compared to the subjects with skeletal class I malocclusion.

Not only sagittal but vertical growth also has its impact on glenoid fossa position. Miranda et al ${ }^{[6]}$ concluded that the distance between glenoid fossa and mandibular condyle is increased in patients presenting with low vertical facial proportions and decreased in patients presenting with high vertical facial proportions. Therefore, patients having both low and high angle vertical dimensions should be included clinically along with radiographical evaluation of glenoid

fossa location to get more constant outcomes. Vertical measurements for the evaluation of the glenoid fossa position are more reliable diagnostic mean as compared to sagittal parameters according to the study conducted by Baccetti et al ${ }^{[11]}$.

It must be highlighted that posteriorly located glenoid fossa resulting in class II malocclusion can be relocated by functional jaw orthopedics ${ }^{[12,13]}$. Functional appliances can cause prominent modifications in the posterior wall of glenoid fossa causing mandibular advancement and condylar growth. These modifications can correct skeletal class II malocclusion due to retrognathic mandible ${ }^{[14,15]}$.

Further studies are needed to establish reference values for measurements involving glenoid fossa position in subjects of various ages and with different dentoskeletal relationships.

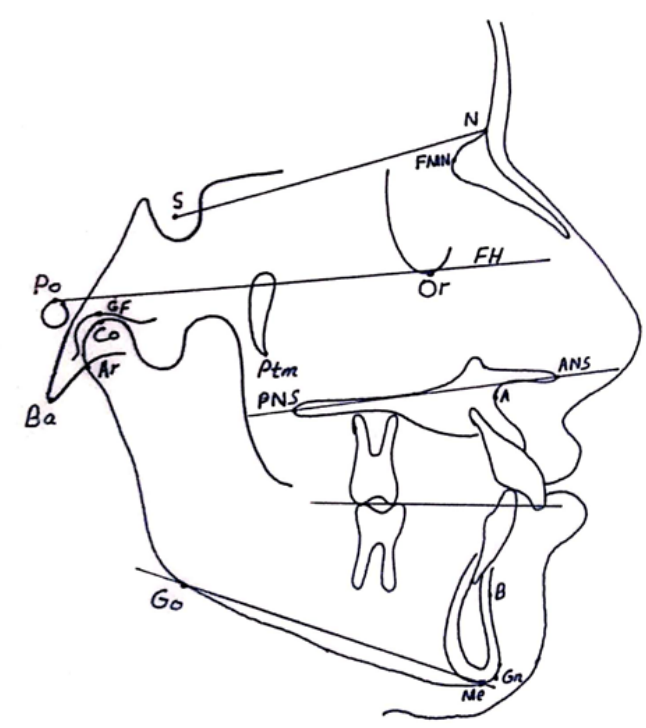

Figure-II: Cephalometric angular and linear measurments ${ }^{[7]}$.

- $\quad<\mathrm{ANB}=<\mathrm{SNA}-<\mathrm{SNB}$

- GF-S on FH

- GF-Ptm on FH

- GF-FMN

- $\quad<$ SN-MP 


\section{CONCLUSION}

Position of glenoid fossa in subjects with class II malocclusion is more distal and posterior as compared to the subjects with class I malocclusion. The effective cephalometric measurements for the evaluation of glenoid fossa position are GF-S on FH, GF-Ptm on FH and GF-FMN.

\section{ACKNOWLEDGMENT: None.}

GRANT SUPPORT \& FINANCIAL DISCLOSURE:

None.

CONFLICT OF INTEREST: All authors declare no conflict of interest.

\section{REFERENCES:}

1. Nurmuhayannah, Erna Sulistyawati, Nazruddin. The changes of position and angulation of condyle mandible before and after orthodontic treatment in class II malocclusion by using Cephalometric Radiograph. Advances in Health Science Research:2017;8: 208210. Doi: 10.2991/idcsu-17.2018.54

2. Cheib Vilefort PL, Farah LO, Gontijo HP, Moro A, Ruellas ACO, Cevidanes LHS et al. Condyle-glenoid fossa relationship after Herbst appliance treatment during two stages of craniofacial skeletal maturation: A retrospective study. Orthodontic and Craniofacial Research. 2019;22(4):345-353. Doi: 10.1111/ ocr.12338.

3. Arvind Mengi, Vijay P Sharma, Paradeep Tandon, Akhil Agarwal, Abhishek Singh. A cephalometric evaluation of the effect of glenoid fossa location on craniofacial morphology. Journal of oral biology and craniofacial research: 2016;6(3):204-212. Doi: 101016/j.jober.2016.06.004

4. Arvind Mengi, Vijay P. Sharma, Pradeep Tandon, Akhil Agarwal. The Glenoid Fossa Location in Cranial Basein Various Skeletal Malocclusions. Journal of Dental Herald:2015; 2(2):6-10.

5. Qadir M, Mushtaq M, Kalgotra S. Diagnostic significance of Glenoid fossa position: A cephalometric study. IOSR Journal of Medical and Dental Sciences.2017;16(12):61-65. Doi: 10.9790/08531612036165

6. Arieta-Miranda JM, Silva-Valencia M, Flores-Mir C, Paredes-Sampen NA, Arriola-Guillen LE. Spatial analysis of condyle position according to sagittal skeletal relationship, assessed by cone beam computed tomography. Progress in Orthodontics. 2013;14:36. Doi: 10.1186/2196-1042-14-36.

7. Giuntini V, De Toffol L, Franchi L, Baccetti T. Glenoid fossa position in class II malocclusion associated with mandibular retrusion. Angle Orthodontics: 2008;78(5):808-812. Doi:10.2319/073007-353.1

8. Arkan M.A, Al-Azzawi, fakhri A. The position of glenoid fossa in different skeletal patterns and its relation to the functional occlusal plane. Journal of Baghdad College of Dentistry: 2010;22(2):81-86.
9. Nagaraj K, Roopa Jatti, Sujala Ganapati Durgekar. Evaluation of morphology and position of glenoid fossa in class I and class II malocclusion. Indian Journal of Orthodontics and Dentofacial Research: 2017;2(4)160165. Doi: 10.18231/2455-6785.2016.0006

10. Droel R, Isaacson RJ. Some relationships between the glenoid fossa position and various skeletal discrepancies. American Journal of Orthodontics:1972; 61:64-78. Doi:101016/0002-9416(72)90177-7

11. Baccetti $\mathrm{T}$, Antonini A, Franchi L, Tonti M, Tollaro I. Glenoid fossa position in different facial types: a cephalometric study. British Journal of Orthodontics:1997; 24(1):55-59. Doi:10.1093/ ortho/24.1.55

12. Ivorra-Carbonell L, Montiel-Company JM, AlmerichSilla JM, Paredes-Gallardo V, Bellot-Arcís C. Impact of functional mandibular advancement appliances on the temporomandibular joint - a systematic review. Medicinia Oral, Patologia oral Y Cirugia Bucal.2016; 21(5):565-572. Doi: 10.4317/medoral.21180.

13. Kyburz KS, Eliades T, Papageorgiou SN. What effect does functional appliance treatment have on the temporomandibular joint? A systematic review with meta-analysis. Progress in Orthodontics. 2019;20(1):3236. Doi; https://doi.org/10.1186/s40510-019-0286-9

14. Wang S, Ye L, Li M, Zhan H, Ye R, Li Y et al. Effects of growth hormone and functional appliance on mandibular growth in an adolescent rat model. Angle Orthodontics. 2018; 88(5):624-631. Doi: 10.2319/120417-829.1.

15. Feres MF, Alhadlaq A, El-Bialy T. Adjunctive techniques for enhancing mandibular growth in Class II malocclusion. Medical Hypotheses Journal. 2015;84(4):301-304. Doi: 10.1016/j.mehy.2015.01.012.

Authors Contribution:

Rabia Tabassum: Designed the study, collected the data, Interpretation of data for the work.

Nazish Amjad: Substantial contributions to the conception. Faiza Malik: Acquisition \& data analysis. 